\title{
Índices para a representação da paisagem como apoio para levantamento pedológico em ambiente de geoprocessamento
}

\author{
Venina Prates $^{1}$, Luiz C. de P. Souza ${ }^{2} \&$ Jairo C. de Oliveira Junior ${ }^{3}$
}

\section{RESU M O}

0 mapeamento de solos tem ganhado destaque dentro da comunidade científica, pois, à medida em que a preocupação com o meio ambiente aumenta, ocorre a necessidade de se entender cada vez mais a distribuição dos solos na paisagem, tal como seu potencial e limitações de uso. Desta forma, o principal objetivo do trabalho foi aplicar índices de representação da paisagem com o apoio de geoprocessamento, suporte na delimitação dos diferentes compartimentos da paisagem. Foram utilizados índices primários Altitude above channel network (AACN) e secundários Channel network base level (CN BL, Multiresolution índex of valley bottom flatness (M RVBF) e W etness índex (ITW ), cujo estudo foi a Fazenda Experimental Canguiri, no município de Pinhais, região Metropolitana de Curitiba. Para correlacionar os atributos químicos e granulométricos por grupo de amostragem, totalizando 17 pontos (Sugamosto, 2002) foi gerada, no Software Statistica, uma matriz de correlação linear simples (Pearson) com os índices de representação da paisagem, os quais foram não apenas utilizados na análise de agrupamentos mas também eficientes no apoio do mapeamento dos solos, a nível de subordem do Sistema Brasileiro de Classificação de Solo.

Palavras-chave: modelagem numérica do terreno, índices de representação da paisagem, análise de agrupamentos

\section{Indices representing landscape as a support for pedological survey in GIS environment}

\begin{abstract}
Mapping of soil has been highlighted in the scientific community, because as alertness about the environment increases, it is necessary to understand more and more about the distribution of the soil in the landscape, as well as its potential and its limitations for the use. In that way the main aim of this study was to apply indices representing landscape with the use of geoprocessing to give support in the delimitation of different compartments of landscape. Primary indices used were altitude above channel network (AACN) and secondary channel network base level (CNBL), multiresolution index of valley bottom flatness (MRVBF) and W etness index (ITW), having as object of study the Canguiri Experimental Farm, located in Pinhais, Curitiba's Metropolitan region. To correlate the chemical attributes and granulometric ones in sampling groups, totalizing 17 points (Sugamosto, 2002), a matrix of a simple linear correlation (Pearson) with the indices of the landscape were generated in the Software Statistica. The conclusion is that the indices representing the landscape used in the analysis of groupings were efficient as support to map soil at the level of suborder of Brazilian Soil Classification System.
\end{abstract}

Key words: Soil numerical modellling, representation indices of the landscape, analysis of groupings

\footnotetext{
${ }^{1}$ Depto. de Geografia com ênfase em Geotecnologias/UTP, CEP 82010-330, Curitiba, PR. E-mail: venina.prates@utp.br

2 Depto. de Solos e Eng. Agrícola/UFPR, CEP 80035-050, Curitiba, PR. E-mail: Icsouza@ufpr.br

Depto. de Solos e N utrição de Plantas/U SP, CEP 13418-900, Piracicaba, SP. E-mail: jairo@agronomo.eng.br
} 


\section{INTRODUÇÃO}

Atualmente, sempre que o mapeamento de solos tem ganhado destaque dentro da comunidade científica, a preocupação com o meio ambiente aumenta e então surge a necessidade de se entender cada vez melhor a distribuição dos solos na paisagem, a variabilidade de seus atributos, seu potencial e suas limitações de uso (Gilo \& Etzelmuller, 2009; Hancock et al., 2010; Oliveira Júnior et al., 2010; 2011).

Portanto, o estudo de índices de representação da paisagem passou a ser mais uma alternativa de avaliação e interpretação da paisagem, sobretudo após o desenvolvimento de computadores potentes e rotinas mais eficazes. Porém, para que esses parâmetros descrevam a variabilidade dos solos na paisagem torna-se oportuno o desenvolvimento de modelos e métodos específicos (Florinsky et al., 2002).

Vários autores mostram a relação relevo solos, dentre os quais se destacam: Cunha et al. (2005), Campos et al. (2006; 2007), que identificaram superfícies geomórficas para estabelecer e separar as áreas naturais e relativamente homogêneas com a declividade do terreno; entretanto, apenas os índices de representação da paisagem não são suficientes para sua delimitação, pois se sabe que a ocorrência dos solos é produto de um conjunto de fatores os quais, atuando sistematicamente, favorecem o desenvolvimento de determinados solos.

Ressalta-se que o relevo é um elemento de grande influência no desenvolvimento dos solos na paisagem, tal como sua percepção nas imagens e sua relação com os limites da unidade de mapeamento, sendo assim significativos como descrito em Goosen (1968).

O índice topográfico de umidade (ITW) caracteriza as zonas de saturação de água superficial e o conteúdo de água nos solos (Pei et al., 2010). Buscando estabelecer uma correlação entre a umidade real dos solos e o índice topográfico de umidade identificou-se que para solos bem drenados, o índice varia entre 4 e 5 , em solos moderadamente drenados ele varia entre 5 e 7 e em solos mal-drenados, os índices oscilam entre 7 e 12. Essas condições de umidade também estão associadas à espessura dos solos, ao grau estrutural e à permeabilidade (Lin et al., 2006).

O índice Multiresolution Index of Valley Bottom Flatness (MRVBF), define e distingue os fundos de vale de encostas em diferentes escalas e combina diferentes paisagens em um único índice (Wang et al., 2010). Para Gallant \& Dowling (2003) o índice MRVBF foi projetado especificamente para mapear áreas de deposição na paisagem. Valores inferiores a 0,5 são considerados superfícies de erosão e valores superiores a 0,5 são superfícies de deposição (Mckenzie \& Gallant, 2007).

A Altitude Above Channel Network (AACN), é a distância vertical a partir da rede de drenagem expressa em metro (Möller et al., 2012); portanto, a altitude é diferente em cada ponto em relação à drenagem, estimando a energia potencial do terreno (Romão, 2006).

AAACN também foi utilizada para a delimitação de classes de solo em que os baixos valores caracterizam superfícies mais próximas do nível do canal, o acúmulo de sedimentos e a proximidade do lençol freático. Os valores médios indicam processos de transferência de material das encostas e os valores elevados foram encontrados em topo de morros.

Para determinar o índice Channel Network Base Level (CNBL) considera-se a distância vertical até o nível do canal de base da rede local, utilizando-se a Modelagem Numérica do Terreno (MNT) como fase inicial (Hansen et al., 2009). Para Bock \& Kothe (2008), o CNBL é essencial para a previsão da profundidade de solos hidromórficos influenciados pelo escoamento de águas subsuperficiais e subterrâneas.

Slope se refere à inclinação da superfície do terreno em relação à inclinação horizontal (Câmara et al., 1996). Assim, solos que ocorrem em relevos mais íngremes sofrem rejuvenescimento por meio de processos erosivos (Sousa Júnior \& Demattê, 2008).

Profile Curvature é a taxa de variação do aspecto ao longo da curva de nível e caracteriza mudanças na velocidade do fluxo da água e processos relacionados ao transporte de sedimentos. Valores positivos devem corresponder a terrenos convexos, valores negativos a terrenos côncavos e valores nulos a terrenos retilíneos (Valeriano, 2008).

Plan Curvature se relaciona à taxa de variação da declividade ao longo das curvas de nível e mede a propensão da água convergir ou divergir sempre que atravessa o terreno. Valores positivos correspondem a terrenos divergentes, negativos a terrenos convergentes e nulos a terrenos planares (Valeriano, 2008).

Em função do exposto é possível perceber que os índices de representação da paisagem se relacionam com os atributos do solo e ao grau de desenvolvimento dos solos. Desta forma, o objetivo do presente trabalho foi avaliar a aplicação dos índices de representação da paisagem como apoio na delimitação dos diferentes compartimentos da paisagem e sua posterior aplicação no mapeamento de solos.

\section{Material e MÉTODos}

A área se localiza na região metropolitana de Curitiba, no município de Pinhais, Paraná (Figura 1), situada entre as coordenadas UTM, datum Córrego Alegre, fuso 22: 685.000 $\mathrm{mW}, 690.000 \mathrm{~mW}, 7.180 .000 \mathrm{mS}, 7.191 .000 \mathrm{mS}$, totalizando uma área de 444,53 ha.

O substrato geológico da área se relaciona com a formação Guabirotuba, composta de argilitos, seguindo-se de arcósios e

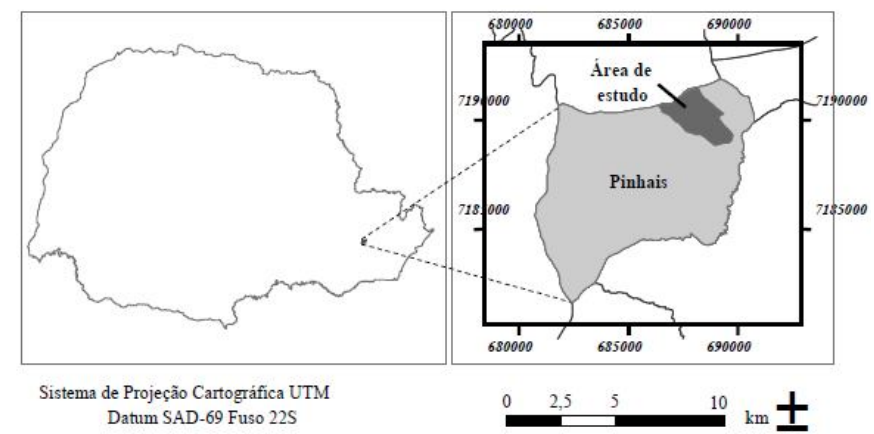

Figura 1. Localização da área de estudo: Fazenda Experimental Canguiri (UFPR) no Município de Pinhais, PR 
depósitos rudáceos, além de pequenos horizontes de caliche (Salamuni \& Stellfeld, 2001). Predominam as formas de relevo suave ondulado a ondulado e, de maneira geral, as atividades de uso e cobertura do solo são agrossilvopastoris desenvolvidas em remanescentes naturais de estepes entremeadas por capões próximos aos cursos d'água (Curcio et al., 2006).

As cartas planialtimétricas da Região Metropolitana de Curitiba - COMEC (1976) foram utilizadas como base cartográfica,em escala 1:10.000, com equidistância entre curvas de nível de $5 \mathrm{~m}$. As curvas foram digitalizadas e posteriormente interpoladas para a geração do modelo numérico do terreno MNT, tendo como interpolador B-Spline Approximation do software System for Automated Geoscientific Analyses (SAGA, 2005).

De posse do modelo numérico do terreno, foram gerados os índices de representação da paisagem, primários e secundários (SAGA, 2005), os quais aparecem discriminados na Tabela 1.

Tabela 1. Métodos para geração dos Índices de Representação da Paisagem

\begin{tabular}{lr}
\hline \multicolumn{1}{c}{$\begin{array}{c}\text { Índices de representação } \\
\text { da paisagem }\end{array}$} & \multicolumn{1}{c}{ Método } \\
Altitude Above Channel Network & Olaya \& Conrad, 2008 \\
Analytical hills had & Olaya \& Conrad, 2003 \\
Aspect & Zevenbergen \& Thrne, 1987 \\
Catchment área & Olaya \& Conrad, 2008 \\
Channel network base level & Olaya \& Conrad, 2008 \\
Convergence index & Kothe \& Lehmeier, 1993 \\
Curvature & Zevenbergen \& Thrne, 1987 \\
Curvature classication & Dikau, 1988 \\
Multiresolution index of valley bottom & Olaya \& Conrad, 2006 \\
flatness & Zevenbergen \& Thrne, 1987 \\
Plan curvature & Zevenbergen \& Thrne, 1987 \\
Profile curvature & Olaya \& Conrad, 2008 \\
LS-factor & Zevenbergen \& Thrne, 1987 \\
Slope & Olaya \& Conrad, 2008 \\
Stream Power & Olaya \& Conrad, 2008 \\
Watershed subbasins & Olaya \& Conrad, 2008 \\
Wetness índex &
\end{tabular}

Os dados químicos e granulométricos dos horizontes A e B dos solos foram extraídos do trabalho de Sugamosto (2002). Desses, dezessete pontos foram selecionados e separados em quatro grupos de amostragem, distribuídos em diferentes posições da paisagem (Figura 2). Os referidos pontos foram submetidos à matriz de correlação linear simples (Pearson) no software Statistica (Statsoft, 2007), com a intenção de se identificar os índices de representação da paisagem que tem correlação com o maior número de atributos.

Os índices de representação da paisagem que apresentaram correlação com um número maior de atributos do solo foram: Channel Network Base Level-CNBL, Altitude Above Channel Network - AACN, Multiresolution Index of Valley Bottom Flatness - MRVBF, os quais foram submetidos à análise de agrupamento, pelo método da Mínima Distância Euclidiana.

Verificou-se, após a aplicação da análise de agrupamentos, que os fundos de vale não estavam bem discriminados e, desta forma, haveria problemas para a identificação dos solos das planícies aluviais. Portanto, foi incluído, na análise de

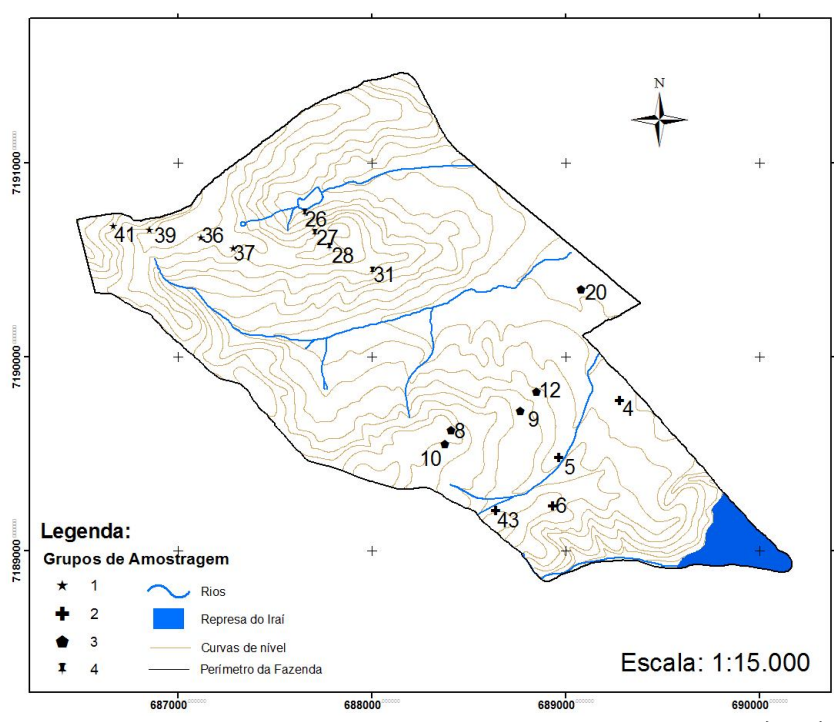

Figura 2. Localização dos G rupos de A mostragem (G A) para a realização da matriz de correlação

agrupamentos, o índice topográfico de umidade (ITW), o qual ajudou na discriminação de tal compartimento da paisagem, mesmo que o produto da análise de agrupamento tenha apresentado polígonos que possuem pequena expressão cartográfica; em consequência, foram descartados na edição final da compartimentalização da paisagem.

Os solos foram classificados no segundo nível categórico do Sistema Brasileiro de Classificação de Solos (EMBRAPA, 2006), com base nos dados analíticos extraídos de quarenta e nove pontos do trabalho de Sugamosto (2002).

Para os agrupamentos que não apresentaram pontos de amostragem, as unidades taxonômicas foram estimadas por extrapolação, considerando-se sempre, inicialmente, a paisagem e, posteriormente, os índices de representação da paisagem.

\section{RESULTADOS E DISCUSSÃO}

\section{Matriz de Correlação dos índices de representação da} paisagem com os atributos químicos e granulométricos

Os índices da matriz de correlação linear de Pearson a 95\% de probabilidade mostram que os atributos químicos e granulométricos do solo possuem alta correlação com os índices de representação da paisagem, indicativo de que esses índices podem ser utilizados como apoio na identificação e delimitação das unidades de mapeamento dos solos (Tabela 2).

Os índices ITW e MRVBF (Tabela 2) indicam a concentração do fluxo de água nos solos e definem as áreas de sedimentação e de erosão, respectivamente. Desta forma, podem estar associados aos processos de intemperização dos solos, seja para promover ou impedir seu desenvolvimento, fato já confirmado por Moore et al. (1991; 1993), McKenzie \& Ryan (1999), McKenzie \& Gallant (2007) e Möller et al. (2012), e que estão diretamente relacionados ao fator de formação dos solos, o relevo, citando como referência o modelo de Jenny (1994).

Os índices AACN e CNBL (Tabela 2) estão associados à distância vertical da superfície em relação ao canal de drenagem 
Tabela2. M atriz de Correlação dos índices de representação da paisagem com os atributos químicos e granulométricos dos solos por grupos de amostragem de solos

\begin{tabular}{|c|c|c|c|c|c|c|c|c|c|c|c|c|c|c|}
\hline \multicolumn{15}{|c|}{ Grupos de amostragem } \\
\hline & \multicolumn{2}{|c|}{ G1A } & \multicolumn{2}{|c|}{ G1B } & \multicolumn{2}{|c|}{ G2A } & \multicolumn{2}{|c|}{ G2B } & \multicolumn{2}{|c|}{ G3A } & \multicolumn{2}{|c|}{ G3B } & \multicolumn{2}{|c|}{ G4A } \\
\hline & A & C & A & C & A & $M$ & A & $M$ & I & $M$ & C & I & I & M \\
\hline $\mathrm{pH} \mathrm{H} \mathrm{H}_{2} \mathrm{O}$ & & & $-0,98$ & & & & & $-0,99$ & & & & $-0,89$ & & \\
\hline $\mathrm{pH}_{\mathrm{KCl}}$ & & & $-1,00$ & & & & 1,00 & & & & & & & \\
\hline $\mathrm{Al}^{+3}$ & & & & & & & & & & & & & 1,00 & \\
\hline $\mathrm{H}+\mathrm{Al}$ & & & & & & & & 1,00 & 0,91 & $-0,91$ & & & 1,00 & \\
\hline $\mathrm{Ca}^{+2}+\mathrm{Mg}^{+2}$ & & & $-0,99$ & $-1,00$ & & & & & & $-0,91$ & $-0,93$ & & & \\
\hline $\mathrm{Ca}$ & & & $-0,99$ & $-1,00$ & & & & & & & $-0,94$ & & & \\
\hline K & & & & & & & & & & & $-0,89$ & & & \\
\hline СТC & & & $-0,99$ & $-1,00$ & $-0,95$ & 0,95 & & 0,98 & & & & & & \\
\hline$P$ & & & & & & & & & & & & & & $-1,00$ \\
\hline C & & 1,00 & & & & & & & & & $-0,96$ & & & $-1,00$ \\
\hline$S$ & & & $-0,99$ & $-1,00$ & & & & & & $-0,91$ & $-0,93$ & & & \\
\hline V\% & & & $-0,99$ & $-1,00$ & & & & & & $-1,00$ & & & & \\
\hline $\mathrm{m} \%$ & & & & & & & & & & 0,91 & & & & \\
\hline Argila\% & 0,97 & 1,00 & 0,97 & 0,99 & & & & & & & & & & \\
\hline Areia $\%$ & & $-1,00$ & & $-1,00$ & & & & & & & & & & \\
\hline Silte $\%$ & $-1,00$ & $-1,00$ & $-1,00$ & $-1,00$ & & & & & & & & & & \\
\hline
\end{tabular}

Valores significativos a 5\% de probabilidade; G1A - Grupos de Amostragem: 1 Horizonte A; G1B - Grupos de Amostragem 1 Horizonte B; G2A - Grupos de Amostragem 2 Horizonte A; G2B - Grupos de Amostragem 2 Horizonte B; G3A - Grupos de Amostragem 3 Horizonte A; G3A - Grupos de Amostragem 3 Horizonte B; G4A - Grupos de Amostragem 4 Horizonte A; G4B - Grupos de Amostragem 4 Horizonte B. A - Altitude Above Channel Network (AACN); C - Channel Network Base Level (CNBL); M - Multiresolution Valley Bottom Flatness (MRVBF) e I - índice topográfico de umidade (ITW)

mais próximo ou em relação ao nível de base local, respectivamente. Além desses índices indicarem a proximidade dos canais de drenagem e, por consequência, as condições de drenagem, podem também reportar a energia potencial da água, conforme Romão (2006), a qual ainda atuará na ação dos processos erosivos estando, portanto, associados ao relevo e, em contrapartida, atendem ao proposto por Jenny (1994) confirmado nos índices de representação da paisagem por Moore et al. (1991; 1993), McKenzie \& Ryan (1999) e McKenzie \& Gallant (2007).

A.
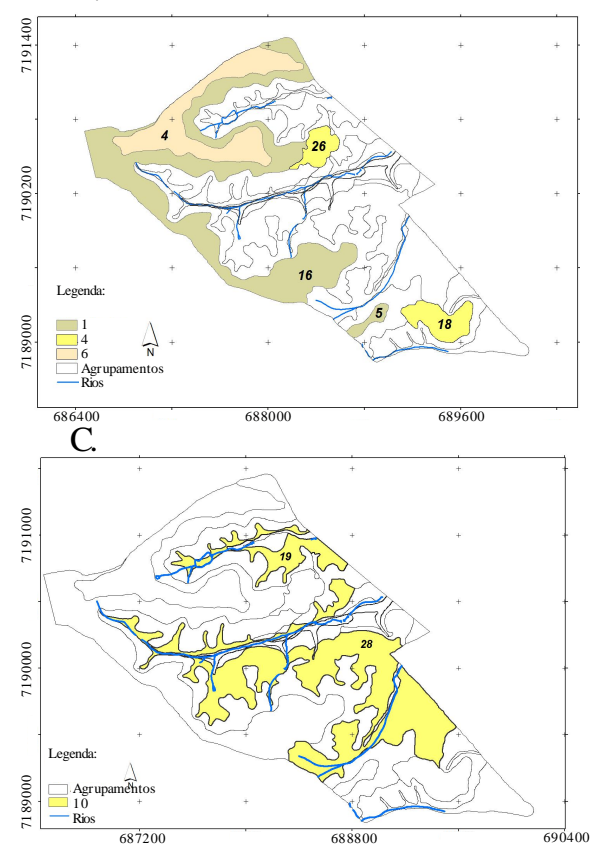

Análise espacial dos índices de representação da paisagem

Os agrupamentos 1, 4 e 6 (Figura 3A) são os que apresentam maior distância vertical em relação ao canal de drenagem, fato que caracteriza solos com boa drenagem; no entanto, a água que se encontra nessas áreas também apresenta uma energia potencial maior, o que pode contribuir para a ação de processos erosivos (Romão, 2006). Apesar disto, os agrupamentos 1 e 6 são característicos de topos de morro, apresentando relevo predominantemente suave ondulado, convexo e divergente. Condições que podem favorecer o maior desenvolvimento dos

B.
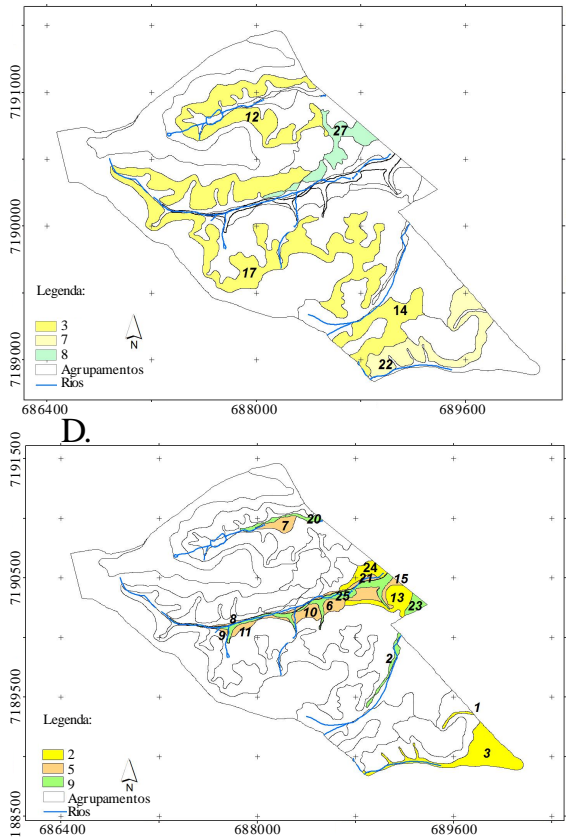

Figura 3. Localização dos agrupamentos 1, 4 e 6 com a identificação dos polígonos 4, 5, 16, 18 e 26 (A); dos agrupamentos 3, 7 e 8 com a identificação dos polígonos 12, 14, 17, 22 e 27 (B); do agrupamento 10 com identificação dos polígonos 19 e 28 (C) e dos agrupamentos 2, 5 e 9 com identificação dos polígonos 1, 2, 3, 6, 7, 8, 9, 10, 11, 13, 15,20, 21, 23, 24 e 25 (D) 
solos, pois a forma do relevo favorece a dispersão do fluxo da água reduzindo o transporte de sedimentos (Valeriano, 2008). Já o agrupamento 4 se diferencia dos anteriores unicamente pelo relevo ondulado. Deve-se ressaltar que esses agrupamentos apresentam ITW baixo e MRVBF superior a 0,5 (Tabela 3), não caracterizando superfícies em que predominem processos erosivos. Assim, os solos tendem a ser menos desenvolvidos em função da declividade a qual favorece a menor infiltração da água e maior escorrimento superficial (Jenny, 1994).

Os agrupamentos 3,7 e 8 (Figura 3B) apresentam distância vertical em relação ao canal de drenagem (AACN) inferior aos agrupamentos anteriores; no entanto, ainda se encontram em posição elevada, o que proporciona solos bem drenados. $\mathrm{O}$ agrupamento se diferencia dos demais por se encontrar em relevo suave ondulado, côncavo e convergente, o que favorece os processos de sedimentação em comparação com os de erosão, fato que pode ser identificado pelo índice MRVBF igual a 0,8 (Mckenzie \& Gallant, 2007).

O agrupamento 7 se caracteriza por uma superfície com predominância de processos erosivos, como pode ser identificado na Tabela 3, em que o valor de MRVBF é de 0,31 diferenciando-se do agrupamento 3 pela presença de relevo côncavo (Gallant \& Dowling, 2003, Mckenzie \& Gallant, 2007).
O agrupamento 10 (Figura 3C) possui relevo suave ondulado, côncavo e convergente, fato passível de favorecer a concentração do fluxo superficial e subsuperficial da água e pode ser evidenciado pelos índices ITW próximos de 9 e MRVBF maiores que 1,7 (Tabela 3). Esta superfície está mais sujeita a processos deposicionais (McKenzie \& Gallant, 2007). Ressaltase que tais superfícies não podem ser caracterizadas com excesso de água uma vez que a distância vertical em relação aos canais, é elevada.

Os agrupamentos 2, 5 e 9 (Figura 3D) apresentam características de fundo de vale com grande concentração de umidade, típico de solos hidromórficos, pois apresenta distância vertical em relação ao canal de drenagem, variando entre 0,01 a $1,27 \mathrm{~m}$ (Tabela 4). O agrupamento 9 é o que apresenta maior hidromorfismo devido aos altos índices de MRVBF e ITW, o que caracteriza áreas com grande concentração de umidade as quais, por sua vez, se caracterizam pela predominância de processos deposicionais complementados pelo relevo plano, côncavo e convergente (Moore et al., 1993, McKenzie \& Gallant, 2007). O agrupamento 5 possui posição intermediária em relação ao hidromorfismo pois, apesar de apresentar baixa distância vertical em relação ao canal de drenagem e altos índices de MRVBF, o que caracteriza um ambiente onde predominam processos sedimentares, o ITW apresenta valores intermediários entre os agrupamentos 2 e 9 e possui relevo suave ondulado.

Tabela 3. Média e Coeficiente de Variação dos índices de representação da paisagem de cada polígono gerado pela análise de agrupamentos (cluster)

\begin{tabular}{|c|c|c|c|c|c|c|c|c|c|c|}
\hline Cluster & Polígono & AACN & $\mathrm{CV}$ & CNBL & $\mathrm{CV}$ & TWI & CV & MRVBF & CV & D-C-P \\
\hline 1 & $\begin{array}{r}5 \\
16\end{array}$ & $\begin{array}{l}11,46 \\
15,73\end{array}$ & $\begin{array}{r}9 \\
25\end{array}$ & $\begin{array}{l}914,67 \\
918,56\end{array}$ & $\begin{array}{l}0,26 \\
0,57\end{array}$ & $\begin{array}{l}6,40 \\
6,84\end{array}$ & $\begin{array}{l}12 \\
14\end{array}$ & $\begin{array}{l}0,55 \\
0,55\end{array}$ & $\begin{array}{l}9 \\
8\end{array}$ & $\begin{array}{c}\text { SO-DV-CX } \\
\text { O-DV-CX }\end{array}$ \\
\hline 2 & $\begin{array}{r}1 \\
3 \\
13 \\
24 \\
\end{array}$ & $\begin{array}{l}0,48 \\
0,60 \\
0,40 \\
1,27\end{array}$ & $\begin{array}{r}135 \\
112 \\
88 \\
59\end{array}$ & $\begin{array}{l}897,26 \\
888,94 \\
898,91 \\
898,72\end{array}$ & $\begin{array}{l}0,43 \\
0,44 \\
0,15 \\
0,15\end{array}$ & $\begin{array}{l}9,77 \\
9,91 \\
8,92 \\
8,99\end{array}$ & $\begin{array}{r}24 \\
27 \\
11 \\
6\end{array}$ & $\begin{array}{l}1,36 \\
2,80 \\
2,31 \\
2,12\end{array}$ & $\begin{array}{l}14 \\
28 \\
26 \\
24\end{array}$ & $\begin{array}{c}\text { O-CV-CC } \\
\text { SO-CV-CC } \\
\text { P-CV-CC } \\
\text { SO-CV-CC }\end{array}$ \\
\hline 3 & $\begin{array}{l}12 \\
14 \\
17 \\
\end{array}$ & $\begin{array}{l}5,17 \\
6,92 \\
4,87 \\
\end{array}$ & $\begin{array}{l}56 \\
44 \\
58 \\
\end{array}$ & $\begin{array}{l}913,99 \\
912,50 \\
915,46 \\
\end{array}$ & $\begin{array}{l}0,52 \\
0,44 \\
0,59 \\
\end{array}$ & $\begin{array}{l}7,29 \\
7,06 \\
7,24 \\
\end{array}$ & $\begin{array}{l}11 \\
18 \\
12 \\
\end{array}$ & $\begin{array}{l}0,48 \\
0,82 \\
0,56 \\
\end{array}$ & $\begin{array}{r}7 \\
12 \\
8 \\
\end{array}$ & $\begin{array}{c}0-C V-C C \\
0-C V-R \\
0-C V-C C\end{array}$ \\
\hline 4 & $\begin{array}{l}18 \\
26 \\
\end{array}$ & $\begin{array}{l}13,64 \\
12,37 \\
\end{array}$ & $\begin{array}{l}20 \\
19 \\
\end{array}$ & $\begin{array}{l}898,82 \\
906,63 \\
\end{array}$ & $\begin{array}{l}0,55 \\
0,19 \\
\end{array}$ & $\begin{array}{l}6,40 \\
6,61 \\
\end{array}$ & $\begin{array}{l}12 \\
14 \\
\end{array}$ & $\begin{array}{l}0,56 \\
0,57 \\
\end{array}$ & $\begin{array}{l}9 \\
9 \\
\end{array}$ & $\begin{array}{l}0-D V-C X \\
0-D V-C X \\
\end{array}$ \\
\hline 5 & $\begin{array}{r}6 \\
7 \\
8 \\
9 \\
10 \\
11 \\
15 \\
21\end{array}$ & $\begin{array}{l}0,11 \\
0,22 \\
0 \\
0,02 \\
0,15 \\
0,08 \\
0,16 \\
0,21\end{array}$ & $\begin{array}{r}85 \\
142 \\
0 \\
140 \\
97 \\
122 \\
90 \\
112\end{array}$ & $\begin{array}{l}901,23 \\
909,55 \\
908,46 \\
908,67 \\
904,01 \\
907,04 \\
897,71 \\
900,94\end{array}$ & $\begin{array}{l}0,21 \\
0,06 \\
0,02 \\
0,02 \\
0,03 \\
0,21 \\
0,14 \\
0,25\end{array}$ & $\begin{array}{r}10,05 \\
10,68 \\
10,54 \\
9,80 \\
9,09 \\
10,09 \\
9,92 \\
10,32\end{array}$ & $\begin{array}{r}10 \\
9 \\
7 \\
9 \\
12 \\
10 \\
12 \\
7\end{array}$ & $\begin{array}{l}3,83 \\
3,93 \\
3,77 \\
3,94 \\
4,06 \\
4,04 \\
3,58 \\
3,64\end{array}$ & $\begin{array}{l}38 \\
37 \\
36 \\
40 \\
45 \\
40 \\
36 \\
35\end{array}$ & $\begin{array}{c}\text { P-PL-CC } \\
\text { SO-CV-R } \\
\text { SO-CV-CC } \\
\text { SO-CV-CC } \\
\text { P-CV-CC } \\
\text { SO-CV-CC } \\
\text { P-PL-CC } \\
\text { P-CV-CC }\end{array}$ \\
\hline 6 & 4 & 25,01 & 10 & 917,84 & 0,59 & 6,82 & 16 & 0,71 & 10 & SO-DV-CX \\
\hline 7 & 22 & 5,65 & 57 & 897,88 & 0,59 & 6,35 & 18 & 0,31 & 137 & FO-CV-CX \\
\hline 8 & 27 & 4,39 & 48 & 902,76 & 0,21 & 7,47 & 8 & 0,80 & 11 & SO-CV-CC \\
\hline 9 & $\begin{array}{r}2 \\
20 \\
23 \\
25\end{array}$ & $\begin{array}{l}0,05 \\
0,05 \\
0,05 \\
0,01\end{array}$ & $\begin{array}{l}179 \\
240 \\
252 \\
293\end{array}$ & $\begin{array}{l}908,81 \\
907,98 \\
898,85 \\
902,94\end{array}$ & $\begin{array}{l}0,41 \\
0,22 \\
0,08 \\
0,47\end{array}$ & $\begin{array}{l}13,91 \\
14,08 \\
12,68 \\
13,98\end{array}$ & $\begin{array}{r}14 \\
18 \\
8 \\
14\end{array}$ & $\begin{array}{l}3,19 \\
3,43 \\
2,84 \\
3,69\end{array}$ & $\begin{array}{l}23 \\
24 \\
22 \\
26\end{array}$ & $\begin{array}{l}\text { P-CV-CC } \\
\text { P-CV-CC } \\
\text { P-PL-CC } \\
\text { SO-CV-CC }\end{array}$ \\
\hline 10 & $\begin{array}{l}19 \\
28\end{array}$ & $\begin{array}{l}1,64 \\
2,02\end{array}$ & $\begin{array}{l}105 \\
113\end{array}$ & $\begin{array}{l}909,76 \\
910,35\end{array}$ & $\begin{array}{l}0,57 \\
0,74\end{array}$ & $\begin{array}{l}9,35 \\
8,90\end{array}$ & $\begin{array}{l}15 \\
14\end{array}$ & $\begin{array}{l}1,96 \\
1,74\end{array}$ & $\begin{array}{l}21 \\
20\end{array}$ & $\begin{array}{l}\text { SO-CV-CC } \\
\text { SO-CV-CC }\end{array}$ \\
\hline
\end{tabular}

CV: Coeficiente de Variação; D - Declividade do terreno conforme classificação EMBRAPA (P: Plano; SO: Suave ondulado; O: Ondulado; FO: Forte ondulado); C: Plan Curvature (DV: Divergente, CV: Convergente; PL: Planar); P: Profile Curvature (CX: Convexo; CC: Concâvo; R: Retilineo) 
O agrupamento 2 é o que melhor apresenta drenagem entre eles, fato que pode ser identificado pelos índices MRVBF e ITW que são os mais significativos e o relevo, que varia entre plano a ondulado (Tabela 4).

\section{Unidades de mapeamento dos solos}

As unidades taxonômicas identificadas para cada agrupamento atenderam ao segundo nível categórico de Sistema Brasileiro de Classificação de Solos (EMBRAPA, 1995).

O Latossolo Vermelho-Amarelo foi melhor caracterizado pelos índices ITW e MRVBF que indicam boa condição de drenagem e processos erosivos menos intensos, condições que favorecem o desenvolvimento do solo, principalmente quando associado ao relevo suave ondulado, convexo divergente (Valeriano, 2008).

Os Cambissolos Háplicos são caracterizados pelo índice MRVBF predominantemente próximos a 0,5 e com relevo variando entre suave ondulado e ondulado e, preferencialmente, nas superfícies côncavas e convergentes, com exceção para os agrupamentos 8 e 10, em que o índice MRVBF assume valores maiores que 0,5 caracterizando menor ação de processos erosivos (Gallant \& Dowling, 2003).

As unidades de mapeamento caracterizadas por solos hidromórficos foram perfeitamente caracterizadas pelos índices ITW, MRVBFe AACN, ao indicarem a possibilidade de acúmulo de água devido à baixa distância vertical em relação ao canal de drenagem (AACN) e altos índices de ITW maior que 8, associados à prevalência de processos de sedimentação descritos pelos altos valores de MRVBF (McKenzie \& Gallant, 2007).

\section{CONClusõES}

1. As matrizes de correlação foram determinantes para se escolher, entre os 16 índices de representação da paisagem, aqueles que foram eficientes no apoio da delimitação das unidades de mapeamento (AACN, CNBL, MRVBF e ITW).

2. Entre os índices de representação da paisagem o CNBL foi o menos eficiente para caracterizar as particularidades do solo e paisagem.

3. A declividade e a forma do relevo foram significativas na discriminação das diferentes unidades de mapeamento.

4. Os valores superiores a 8 do ITW serviram como apoio para classificar solos com caráter hidromórfico.

5. Os índices de representação da paisagem podem auxiliar na discriminação e delimitação das unidades de mapeamento de solos.

\section{LITERATURA CITADA}

Bock, M; Kothe, R. Predicting the Depth of hydrologic soil characteristics hamburger beiträge zur physischen geographie und landschaftsökologie -Heft 19, 2008.
Câmara, G.; Souza, R. C. M.; Freitas, U. M.; Garrido, J. Spring: Integrating remote sensingand GIS by object-oriented data modelling. Computers \& Graphics, v.20 p.395-403,1996.

Campos, M. C. C.; Campos, M. C. C.; Cardozo, N. P.; Marques, J. J. Relações solo paisagem em uma litossequência arenitobasalto na região de Pereira, SP. Revista Brasileira de Ciência do Solo, v.31, p.519-539, 2007.

Campos, M. C. C.; Cardozo, N. P.; Marques, J. J. Modelos de paisagem e sua utilização em levantamentos pedológicos. Revista de Biologia e Ciências da Terra, v.6, p.104-114, 2006.

COMEC - Coordenadoria da Região Metropolitana de Curitiba, Estado do Paraná. Cartas planaltimétricas, 1:50.000, 1976.

Cunha, P.; Marques, J. J.; Curi, N.; Pereira, G. T.; Lepsch, I. F. Superfícies geomórficas e atributos de latossolos em uma sequência arenítico-basáltico da região de Jabotical (SP). Revista Brasileira de Ciência do Solo, v.29, p.81-90, 2005.

Curcio, G. R.; Bonnet, A.; Pestana, D.; Souza, L.; Socher, L. G. Compartimentação toposequencial e caracterização fitossociológica de um campão de floresta Ombrófila Mista. Revista Floresta, v.36, p.361-369, 2006.

EMBRAPA - Empresa Brasileira de Pesquisa Agropecuária. Procedimentos normativos de levantamentos pedológicos. Brasília: Serviço de Produção de Informação, 1995. 101p.

EMBRAPA - Empresa Brasileira de Pesquisa Agropecuária. Centro Nacional de Pesquisa de Solos. Sistema brasileiro de classificação de solos. 2.ed. Rio de Janeiro: Embrapa Solos, 2006. 412p.

Florinsky, I. V.; Eilers, R. G; Manning, G R.; Fuller, L. G. Prediction of soil properties by digital terrain modeling. Environmental Modelling \& Software, v.17, p.295-311, 2002.

Gallant, J. C.; Dowling, T. I. A multi-resolution index of valley bottom flatness for mapping depositional areas. Water Resource Research, v.39, p.1347, 2003.

Gilo, M. D.; Etzelmuller, B. Spatial prediction of soil classes using digital terrain analysis and multinomial logistic regression modeling integrated in GIS: Examples from Vestfold County, Norway. Catena, v.77, p.8-18, 2009.

Goosen, D. Interpretacion de fotos aereas y su importancia em levantamiento de suelos. Rome: Organizacion Naciones Unidas y para la Agricultura y la Alimentacion. 1968. 56p. Boletim sobre Suelos, 6

Hancock, G. R.; Murphy, D.; Evans, K. G. Hillslope and catchment scale soil organic carbon concentration: An assessment of the role of geomorphology and soil erosion in an undisturbed environment. Geoderma, v.155, p.36-45, 2010.

Hansen, M. K.; Brown, D. J.; Dennison, P. E.; Graves, S. A.; Bricklemyer, R. S. Inductively mapping expert-derived soillandscape units within dambo wetland catenae using multispectral and topographic data. Geoderma, v.150, p.7284, 2009.

Jenny, H. Factors of soil formation-a system of quantitative pedology. New York: McGraw-Hill, 1994. 288p.

Lin, H. S. ; Kogelmann, W. ; Walker, C. ; Bruns, M. A. Soil moisture patterns in a forested catchment: A hydropedological perspective. Geoderma, v.131, p.345-368, 2006.

Mckenzie, N. J.; Gallant, J. C. Digital Soil Mapping With Improved Environmental Predictors and Models of Pedogenesis. In: Lagacherie, P.; Mcbratney, A. B.; Voltz, M. Developments in Soil Science, v.31, p.327-349, 2007. 
Mckenzie, N. J.; Ryan, P. Spatial prediction of soil properties using environmental correlation. Geoderma, v.89, p.67-94, 1999.

Möller, M.; Koschitzki, T.; Hartmann, K.; Jahn, R. Plausibility test of conceptual soil maps using relief parameters. Catena, v.88, p.57-67, 2012.

Moore, I. D.; Gessler, P. E.; Nielsen, G. A.; Peterson, G. A. Soil attribute prediction using terrain analysis. Soil Science Society American Journal, v.57, p.443-452, 1993.

Moore, I. D.; Grayson, R. B.; Ladson, A. R. Digital terrain modelling: A review of hydrological, geomorphological, and biological applications. Hydrology Processes, v.5, p.3-30, 1991.

Oliveira Júnior, J. C.; Souza, L. C. P.; Melo, V. F. Variabilidade de atributos físicos e químicos de solos da Formação Guabirotuba em diferentes unidades de amostragem. Revista Brasileira de Ciência do Solo, v.34, p.1491-1502, 2010.

Oliveira Júnior, J. C.; Souza, L. C. P.; Melo, V. F.; Rocha, H. O. No Prelo. Variabilidade espacial de atributos mineralógicos de solos da Formação Guabirotuba, região Metropolitana de Curitiba, PR. Revista Brasileira de Ciência do Solo, v.35, p.1481-1490, 2011.

Pei, T.; Qin, C.; Zhu, A.; Yang, L.; Luo, M.; Li, B.; Zhou, C. Mapping soil organic matter using the topographic wetness index: A comparative study based on different ow-direction algorithms and kriging methods. Ecological Indicators, v.10, p.610-619, 2010.
Romão, P. A. Modelagem de terreno com base na morfometria e em sondagens geotécnicas - Região de Goiânia, GO. Brasília: Universidade de Brasília, 2006. 192p. Tese Doutorado

SAGA - System for Automated Geoscientific Analyses. Version: 2.0.2. (2005). http://www.saga-gis.org. 19 Jan. 2010.

Salamuni, E.; Stellfeld, M. C. Banco de dados geológicos georeferenciados da Bacia Sedimentar de Curitiba (PR) como base de sistema de informação geográfica (SIG). Boletim Paranaense de Geociências, v.49, p.21-32, 2001.

Sousa Júnior, J. G. A.; Demattê, J. A. M. Modelo digital de elevação na caracterização de solos desenvolvidos de basalto e material arenítico. Revista Brasileira de Ciência do Solo, v.32, p.449-456, 2008.

Statsoft, Inc. (2007). Statistica (data analysis software system), version 8.0. http://www.statsoft.com. 5 Jan. 2010.

Sugamosto, M. L. Uso de técnicas de geoprocessamento para elaboração do mapa de aptidão agrícola da adequação de uso do centro de estações experimentais do Canguiri, Município de Pinhais - Paraná. Curitiba: Universidade Federal do Paraná, 2002. 133p. Dissertação Mestrado

Valeriano, M. M. Topodata: Guia de utilização de dados geomorfométricos locais. São José dos Campos: INPE, 2008. 44p.

Wang, D.; Laffan, S. W.; Liu, Y.; Wu, L. Morphometric characterisation of landform from DEMs. International Journal of Geographical Information Science, v.24, p.305326,2010 . 